\title{
Und wie sind Sie gelabelt?
}

_ Neulich habe ich eine Physiotherapiepraxis im Süden der Republik gesucht. Via Internet. Ich habe Praxen entdeckt, in der zwölf Mitarbeiter als alles mögliche, nur nicht als Physiotherapeuten vorgestellt wurden. Manual-, Bobath-, Brügger-, Dorn-, PNF- und ... xyz-Therapeuten. Nicht einfach für Patienten.

_ Wie wäre es mit diesem Versuch: Bezeichnen Sie sich 2011 einfach mal konsequent als Physiotherapeutin bzw. Physiotherapeut. Widerstehen Sie der Neigung, Ihrin der Weiterbildung erworbenes „Zusatz-Label“ zu Ihrer Berufsbezeichnung zu machen. Sie nennen sich - nicht ohne Stolz - xyz-Therapeut? Okay. Das unterscheidet Sie von Ihren Kollegen und ermöglicht es diesen, Sie einzuordnen. Dagegen spricht nichts. Doch in der Kommunikation mit Nicht-Physiotherapeuten ist eine derartige Differenzierung aus meiner Sicht zu hinterfragen.

_ Wir alle leisten gute Öffentlichkeitsarbeit für die Physiotherapie, wenn wir uns nach außen konsequent als Physiotherapeuten bezeichnen. Das stärkt uns als Gruppe. Eine gemeinsame Sprache stabilisiert die Berufsidentität und fördert den Gemeinschaftssinn. In einem ausufernden Gesundheitssystem mit unzähligen Gesundheits-, Wellness- und Weiß-Gott-was-für-Angeboten ist Stärke kein Luxus.

_ Woher kommt die Neigung, sich stärker mit zum Beispiel einer Zusatzausbildung zu identifizieren als mit der gesamten Physiotherapie? Wissen Sie es? Richtig kritisch wird es aus meiner Sicht, wenn unsere Identifikation mit Teilbereichen der Physiotherapie, also beispielsweise mit Konzepten, so stark ist, dass es die Weiterentwicklung der Physiotherapie bremst. Zunehmend keimt Evidenz aus internationaler und nationaler Forschung, und die Erkenntnisse decken sich nicht immer mit unseren Erfahrungswerten, Denkmodellen und Glaubenssätzen. Was tun, wenn man doch ein leidenschaftlicher xyz-Therapeut ist und diesen Status nicht verlieren will? Die Forschungsergebnisse ignorieren, in Frage stellen oder einfach assimilieren? Das wäre sicher nicht im Sinne der „Erfinder“ und auch nicht im Sinne der Physiotherapie.

Das physiopraxis-Team und ich wünschen Ihnen ein gutes neues Jahr, Ihre

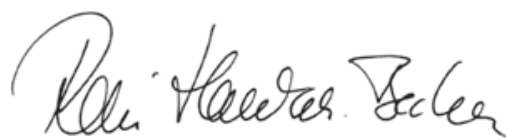

Physiotherapie: ein gutes Label!

\section{PHYSIOBONUS}

physiobonus heißen die Gewinnspiele in physiopraxis.

Wer teilnehmen möchte, nutzt das Formular unter www.thieme.de/ physioonline in der Rubrik physioexklusiv. Und das gibt es in dieser Ausgabe zu gewinnen:

Kursplatz

1 Einführungs-Erlebnistag „Aqua-

Relax, WATSU und WATA“ Seite 55

Bücher

3-mal „Bin Knüller“ $\quad$ Seite 25

1-mal „Manuelle

Triggerpunkt-Therapie“ Seite 29

und außerdem

3 Entscheidungswürfelsets Seite 43

1 Jahresabonnement „Mensch und Pferd international“ $\quad$ Seite 51 